\title{
Gamified Augmented Reality Training for An Assembly Task: A Study About User Engagement
}

\author{
Diep Nguyen \\ UniTyLab \\ Heilbronn University \\ Max-Planck-Strasse 39 \\ 74081 Heilbronn, Germany \\ Email: diep.nguyen@hs-heilbronn.de
}

\author{
Gerrit Meixner \\ UniTyLab \\ Heilbronn University \\ Max-Planck-Strasse 39 \\ 74081 Heilbronn, Germany \\ Email: gerrit.meixner@hs-heilbronn.de
}

\begin{abstract}
Augmented Reality and Gamification are displaying beneficial effects to enhance user experience and performance in many domains. They are widespread across many areas like education, industrial training, marketing, and services. However, the idea of combining the two approaches for an innovative training instrument is fairly new, especially in assembly training. Moreover, learning about the effects of gamification on human, user engagement, in particular, is a complicated subject. There have been several efforts toward this direction, yet the overall situation is still nascent. In this work, we present a gamified augmented reality training for an industrial task and investigate user engagement effect while training with the gamified and the nongamified system. The result shows that people perform better and engage to a greater degree in the gamified design.
\end{abstract}

\section{INTRODUCTION}

A UGMENTED Reality (AR) is growing stronger than ever. Market research predicts a 70 to 75 billion revenue for AR by 2023 [5] and by 2019 AR for training, in particular, will take place in $20 \%$ of large enterprise businesses [6]. AR is the novel technology which superimposes virtual objects upon the real world subjects or environment while enabling real-time interactions [1]. In recent years, AR has captured the research interests in many areas such as education and training [2], [13], assembly and production operations [3], [4]. As a result, the outcome of teaching and learning, skill acquisition and development as well as user experience have shown outstanding beneficial effects.

Gamification, on the other hand, is the term for adapting the design elements which commonly characterize entertainment games into other settings but gaming. While the academic world is still debating on the consensus of definition and scope, the benefits that gamification brings are undenialble. It is not uncommon to say that games are addictive, yet beyond entertainment purposes, they are believed to better life in many aspects [9]. Gamification's ultimate goal is to simulate the fun elements that enhance the user experience, improve worker productivity or advance student engagement. Since gamification is often mistaken with the meaning of the "serious game," which is any full-fledged game that used for other purposes exceeding pure entertainment, we limit the work in this paper to the most widely accepted definition of gamification [10]:

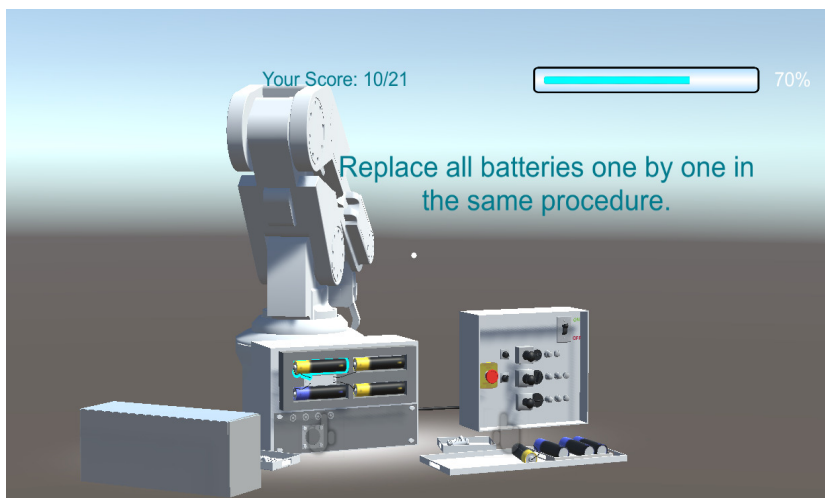

Fig. 1. The GAR design with gamification elements: points, progress bar and signposting.

"Gamification is the use of game design elements in nongame contexts."

Since both AR and gamification already have their certain contribution into the education field, in the context of training especially, it is surprising that gamified AR systems have not been popular for training in the production environment. Accountable for this probably is the fine line between making work fun and making fun of work [7]. Due to the nature of productional work, the misuse of the gamified systems could take away the user's focus attention and result in damages or even injuries. Therefore, here we attempt to form a gamified AR application for an assembly training task following special design requirements for a production environment. Our focus is on the user engagement aspect because it is an important factor contributes to the effectiveness of training.

\section{RELATED WORK}

Although the term "gamification" is relatively new, since around 2003, its applications have already widespread across many industrial as well as scholarly fields. Recently in the Gamification 2020 report, Gartner predicted that gamification in combination with emerging technologies will create a significant impact on several fields including the design of employee performance and customer engagement platform [8]. In this context, there are numerous examples of studies for 
either AR training or gamified training, yet there was hardly any work on the combination of those.

A recent survey of Seaborn et al. [14] provides a good overview of gamification from a Human-Computer-Interaction perspective in both theoretical and practical lights. The work showed that gamification is primarily practiced in the domain of education, e-learning especially. In the theoretical foundations, there was a dynamic movement towards carving the boundaries between gamification and other similar concepts. The applied research, meanwhile, painted a positive-leaning but mixed picture about the effectiveness of gamified systems. Despite usual expectation, similar gamified designs under different settings returned clashing result over user experience along with performance. The reason was believed to be highly context-specific requirements. Furthermore, learning about the effects of gamification on the human is a complicated subject. The overall effort toward this direction is still nascent.

While the gamified system was well accepted in business contexts, it is not necessarily the case in production training, left alone Augmented Reality training. K. Lee [13] showed that $\mathrm{AR}$ for education and training innovation was leaning towards the "serious game" pole while gamification was left outside of the picture. According to Lee, AR games were particularly interested in by both "educators and corporate venues." A roleplaying game for teaching history [11], for example, proved the benefit of enabling students for problem-solving, increasing collaboration and exploration via the virtual identities.

However, whether we like it or not, production training is different from traditional classroom training. When transforming the operational work into a game, a serious game, there will always be a risk of taking the focus away from the task at hand. This is when gamification comes to play as integrating gamification can provide the fun aspect while still keeping the workers' full attention on the operative job [12].

Probably the most well-known gamification in production is a series of works from Korn et al. [15], [16], [17], [12]. The center of his works is to evaluate users' acceptance of gamification in modern production environments. Different designs, "Circles \& Bars" and "Pyramid," were proposed [12]. Both designs were used to visualize work steps as well as their sequences. Color-coded from dark green to yellow, orange and read is employed to indicate user specific time progression. Later on, they were projected into users' working space as an assistive application for impaired individuals. The result indicated a good acceptance level for gamification designs and the "Pyramid" approach was favorable in general. While the study showed a promising outcome, it focused on user acceptance and did not measure the quantitative factor of gamification on task completion time and error rates.

\section{IMPLEMENTATION}

In this section, we present the implementation of the application under study. A process of replacing the battery for a robot arm was implemented based on the instruction manual of the Mitsubishi Industrial Robot RV-2F Series [18]. The application ran on the Microsoft HoloLens [19]. Two

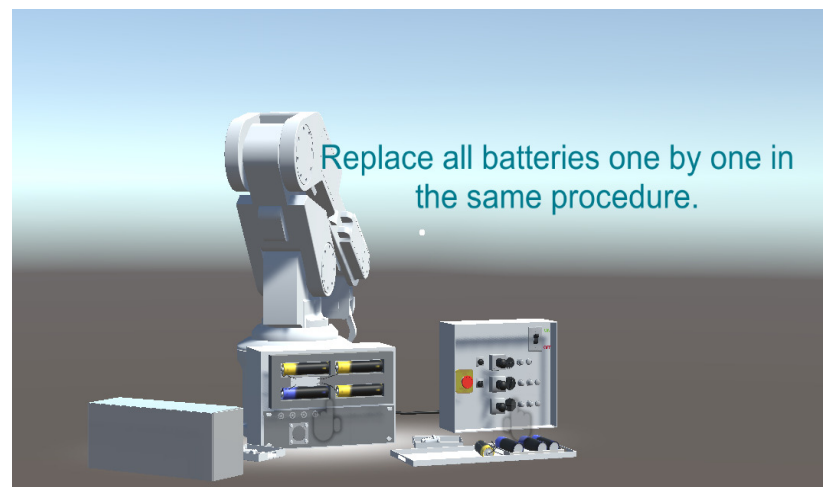

Fig. 2. The NGAR design with no gamification elements. Only text instruction was provided.

prototypes were made, one with the gamification design and the other without. The designs were named Gamification AR (GAR) and Non-Gamification AR (NGAR) according to their characteristics. Due to Microsoft HoloLens small field of view, around 35 degrees, here we provide the user interfaces captured from Unity Editor to showcase the whole scene setup. Figure 1 and Figure 2 illustrate the GAR and NGAR design respectively.

\section{A. The application}

The process for changing the battery was identically built for both prototypes. There were 21 actions made up 10 steps. Disassembling the cover of the battery compartment, for example, included two steps of removing the screws and removing the cover. While removing each of the screws was counted as an action.

For navigating the process, we augmented the instruction text for each step as a head-up display which was always facing the user at the top right corner of the user view. An instruction manager was used to control the flow of text visualization. The requirement from the instruction manual specified that the steps of the process had to be performed in a fixed order that's why only one instruction was displayed at a time. The next instruction triggered when the user carried the current step correctly.

Two main interaction types were used to simulate different interactions. Air tap [19] was used for interacting with static objects (e.g. pressing a button) while we utilized drag and drop for assembling actions (e.g. removing the screw). Similar to the real working space, disassembled objects were designed to be placed at a specific location. For instance, the screws needed to be placed inside a designated tray instead of dropped on the floor.

To simulate a sense of reality, sounds such as robot arm were running or turned off were used.

\section{B. Gamification Design}

The game design elements were implemented only for the GAR version. It allows to isolate and analyze the effect 
of gamified system on the user. This could be reflected by comparing the outcome of the two experiments.

As a result of Korn's investigation [12], gamification in the production environment has its own specific requirements. To avoid resistance from users or the potential of taking away their main focuses, we followed the identified requirements in designing gamified application for production settings. First, "keep the visualization of gamification simple." This focuses mainly on avoiding animation, moving elements and using complex graphical structures. The second and third requirements come together as "avoid explicit interaction with gamification elements" and "support implicit interaction with gamification elements." For that matter, in our designs we did not ask for any user's effort to direct input or reach out to the gamified items.

1) Point System: The point system was built based on users' actions. There was a maximum of 21 points according to 21 actions. Points were rewarded to the user when the action was done. As the first attempt to study the effect of gamification design on user engagement, we did not implement a complex point system with losing points or rewarding extra points at this stage.

2) Progress Bar: While the points were based on actions, progress bar visualized the steps. As stated as one of the requirements, the user interface was intentionally kept simple with only one color. Additional text was in place for indicating the percentage.

3) Signposting: Signposting aims to direct the user in the right direction. While users without background knowledge could be confused with the mechanical part names (e.g. Controller box), signposting highlighted the part corresponding to the currently displayed instruction. It provided the "just-intime" hints for the trainees, especially the totally beginner one.

\section{EXPERIMENT DESIGN}

The experiment was conducted to investigate how gamification in AR training impacts user engagement and performance. The studies for both conditions (GAR and NGAR) took place in the same room at our research laboratory. To avoid the learning effect, we employed the between-group design in which each participant randomly exposed to only one design, either GAR or NGAR.

Due to the fact that Microsoft HoloLens requires specific hand gestures for interaction, the participants were asked if they have experience with this device. In the case of none, the participant used the default HoloLens "Learn gesture" application. This was especially important because the main task could not be carried on without this step. Before the experiment, regardless of the HoloLens experience, we repeated the main information about the interactive gestures to all participants.

Once the participants were confident interacting with the device, the main experiment task proceeded. When the user hit the "Start" button at the first scene of the application, the timer for measuring task completion time was started until the last step completed.
As we focused on the user engagement we used a poststudy questionnaire with the refined User Engagement Scale (UES) [20]. UES is a five-point rating scale: strongly disagree, disagree, neither disagree nor agree, agree and strongly agree, respectively from 1 to 5 point. Given the task was not complicated, the level of fatigue after that was expected not to be high so that we decided to use the UES long form (UES - LF). The UES - LF consists of 30 items covering 4 factors:

1) FA: Focused Attention

2) PU: Perceived Usability

3) AE: Aesthetic Appeal

4) RW: Reward Factor

As constructed in the guide to use of UES, all items were randomized and the indicators (e.g. AE.1) were not visible to the users.

\section{Results}

Most of the participants reported having little or none experience with AR technology, in particular, Microsoft HoloLens, before this experiment. So, a potential novelty effect when initially establishing interaction with new technology might influence the research result. The test population was 22 participants with 11 regarding each condition. Participants ages vary from 18 to 34 years old, 15 male and 7 female subjects. Although some unease and uncertainty were expressed at the beginning, all participants were more certain after the learning gesture phase.

Figure 3 displays that the GAR design was rated better in all sub categories. In general, it was clearly preferred to the NGAR approach. The overall Engagement score was 15.2 $(\mathrm{SD}=1.8)$ in GAR and $13.3(\mathrm{SD}=3.5)$ in NGAR. However, this did not make up a statistically significant difference between the two groups. Table I provides the results in more detail, looking at the average score, standard deviation and also the result of a t-test for both the overall engagement score and its factor.

The standard deviation in the overall user engagement score was much lower in the GAR design $(\mathrm{SD}=1.8)$, versus $\mathrm{SD}=3.5$ in NGAR, which shows that the GAR subjects more homogenously perceived the result throughout the group. This tendency, lower standard deviation, remained true for all four subfactors in the GAR design as shown in Figure 3. On the other side, the opinions of NGAR subjects seem to be more diverse.

Looking at the training performance, the difference regarding average task completion time (in seconds) between the two study conditions is statistically significant. The t-test resulted in $\mathrm{p}<0.032$. The average time was $306.9(\mathrm{SD}=123.2)$ and 439.5 (SD=134.4) for GAR and NGAR groups respectively. This positive outcome probably directly influenced by the signposting design element.

\section{DiscusSiON AND FUtURE WORK}

As a preliminary result, this work demonstrates the potential of gamified AR training for assembly tasks in improving user engagement and performance. Nevertheless, there is a need for 


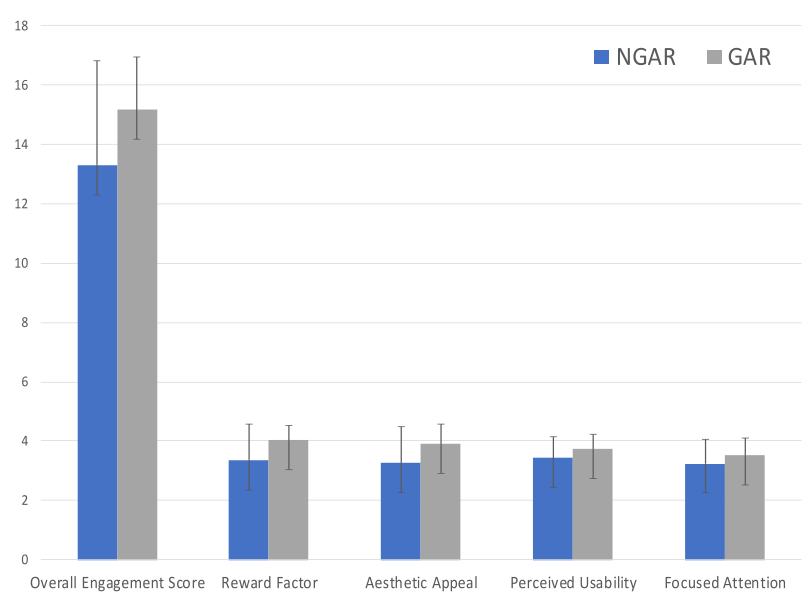

Fig. 3. User Engagement Score as a bar chart with indicated standard deviations.

TABLE I

COMPARISION OF USER ENGAGEMENT SCORE

\begin{tabular}{|l|c|c|c|}
\hline Factor & \multicolumn{2}{|c|}{ Mean Score (SD) } & p value \\
\hline Design & GAR & NGAR & GAR vs. NGAR \\
\hline Focused Attention & $3.5(0.6)$ & $3.2(0.8)$ & $\begin{array}{c}0.418 \\
\text { not significant }\end{array}$ \\
\hline Perceived Usability & $3.7(0.5)$ & $3.4(0.7)$ & $\begin{array}{c}0.281 \\
\text { not significant }\end{array}$ \\
\hline Aesthetic Appeal & $3.9(0.7)$ & $3.3(1.2)$ & $\begin{array}{c}0.162 \\
\text { not significant }\end{array}$ \\
\hline Reward Factor & $4.0(0.5)$ & $3.4(1.2)$ & $\begin{array}{c}0.128 \\
\text { not significant }\end{array}$ \\
\hline Overall Score & $15.2(1.8)$ & $13.3(3.5)$ & $\begin{array}{c}0.153 \\
\text { not significant }\end{array}$ \\
\hline
\end{tabular}

further investigation focusing on both short-term and longterm training effectiveness. A consideration over skills and knowledge acquisition should be taken into account. To serve this goal more complex tasks should be implemented with a higher level of gamification, different training levels and challenges design for individual specific demands for example.

As we focused on the improvement of user engagement in gamified AR training, we did not take in to account the isolated effect of how each game design elements affects the user. As mentioned in the Related Work, gamification design is highly context-specific so that the next important step will be a qualitative study on how the users perceive different design elements and their impacts.

\section{CONCLUSION}

The use of gamification in combination with AR for production training is still new and its potential needs further exploration. In this paper, we developed a gamified training for an assembly task in AR setting and studied its effects on user engagement.

The result showed that the users displayed a higher level of engagement as well as better performance with the support of gamified AR training. The statistical analysis, though, did not indicate a significant difference.

While the implementation of gamification may not yet fully integrate into the training process, this work certainly contributes to the existing knowledge body of gamified AR training for production domain. This research area also needs a greater amount of works to identify its benefits alongside with how to tackle its challenges.

\section{REFERENCES}

[1] T. R. Azuma, “A Survey of Augmented Reality," Presence: Teleoperators and Virtual Environments, vol. 6, no. 4, 1997, pp. 355-385.

[2] M. Billinghurst, H. Kato and I. Poupyrev, "The Magic Book Moving seamlessly between reality and virtuality," IEEE Computers, Graphics and Applications, vol. 21, no. 3, May/June 2001, pp. 2-4.

[3] S. K. Ong , M. L. Yuan and A. Y. C. Nee, "Augmented reality applications in manufacturing: a survey," International Journal of Production Research, vol. 46, 2008, pp. 2707-2742.

[4] W. Friedrich, "ARVIKA: Augmented Reality for Development, Production and Service," The 1st International Symposium on Mixed and Augmented Reality (ISMAR), 2002, pp. 3-4.

[5] For AR/VR 2.0 to live, AR/VR 1.0 must die, DigiCapital, https://www.digi-capital.com/news/2019/01/ for-ar-vr-2-0-to-live-ar-vr-1-0-must-die/. [Retrieved May 2019]

[6] Transform Business Outcomes With Immersive Technology, Smarter with Gartner, https://www.gartner.com/smarterwithgartner/ transform-business-outcomes-with-immersive-technology/. [Retrieved May 2019]

[7] S. Dale, "Gamification: Making work fun, or making fun of work?," Business Information Review, vol. 31, no. (2), 2014, pp. 82-90.

[8] Gamification 2020: What Is the Future of Gamification?, https://www. gartner.com/en/documents/2226015. [Retrieved May 2019]

[9] J. McGonigal, "Reality is broken: Why games make us better and how they can change the world," New York: Penguin, 2011.

[10] S. Deterding, D. Dixon, R. Khaled and L. Nacke, "From Game Design Elements to Gamefulness: Defining Gamification," Proceedings of the 15th International Academic MindTrek Conference: Envisioning Future Media Environments, MindTrek 2011, vol. 11, 2011, pp. 9-15.

[11] K. L. Schrier, "Revolutionizing history education : using augmented reality games to teach histories," Institute of Technology. Dept. of Comparative Media Studies, Massachusetts, 2005.

[12] O. Korn, M. Funk and A. Schmidt, "Design approaches for the gamification of production environments," in Proceedings of the 8th International Conference on PErvasive Technologies Related to Assistive Environments, ACM, New York, NY, USA, 2015, pp. 1-7.

[13] K. Lee, "Augmented Reality in Education and Training," TechTrends, vol. 56, 2012, pp. 13-21, https://doi.org/10.1007/s11528-012-0559-3

[14] K. Seaborn and D. I. Fels, "Gamification in theory and action: A survey," International Journal of Human Computer Studies, vol. 74, 2015, pp. 14-31.

[15] K. Oliver, "Industrial Playgrounds. How Gamification Helps to Enrich Work for Elderly or Impaired Persons in Production," In: Proceedings of the 4th ACM SIGCHI Symposium on Engineering Interactive Computing Systems, New York, 2012, pp. 313-316.

[16] O. Korn, M. Funk, S. Abele, A. Schmidt and T. Hörz, "Contextaware Assistive Systems at the Workplace. Analyzing the Effects of Projection and Gamification," In: PETRA 14 Proceedings of the 7th International Conference on PErvasive Technologies Related to Assistive Environments, ACM, New York, NY, USA, 2014.

[17] O. Korn, M. Funk and A. Schmidt, "Towards a Gamification of Industrial Production. A Comparative Study in Sheltered Work Environments,' In: Proceedings of the 7th ACM SIGCHI Symposium on Engineering Interactive Computing Systems, ACM, New York, NY, USA, 2015.

[18] Mitsubishi Industrial Robot RV-2F Series: Instruction Manual Robot Arm Setup \& Maintenance, www.geva-roboter.at/files/rv-2f_series_ robot_arm_setup__maintenance.pdf. [Retrieved May 2019]

[19] Introduction to the HoloLens, https://msdn.microsoft.com/en-us/ magazine/mt788624.aspx. [Retrieved May 2019]

[20] H. L. O'Brien, P. Cairns and M. Hall, "A practical approach to measuring user engagement with the refined user engagement scale (UES) and new UES short form," International Journal of Human Computer Studies, vol. 112, 2018, pp. 28-39. 